\title{
Chapter 13 \\ Evolutionary Trends in Phenotypic Elements of Seasonal Forms of the Tribe Junoniini (Lepidoptera: Nymphalidae)
}

\author{
Jameson W. Clarke
}

\begin{abstract}
Seasonal polyphenism in insects is the phenomenon whereby multiple phenotypes can arise from a single genotype depending on environmental conditions during development. Many butterflies have multiple generations per year, and environmentally induced variation in wing color pattern phenotype allows them to develop adaptations to the specific season in which the adults live. Elements of butterfly color patterns are developmentally semiautonomous allowing for detailed developmental and evolutionary changes in the overall color pattern. This developmental flexibility of the color pattern can result in extremely diverse seasonal phenotypes in a single species. In this study, we asked the following questions: (a) How do wing phenotype elements such as shape and pattern vary between seasonal forms? (b) Can this variation be explained phylogenetically? (c) If so, what are the various pattern development strategies used to achieve crypsis in the dry season form? To answer these questions, we used high-resolution images to analyze pattern element variation of 34 seasonally polyphenic butterfly species belonging to the tribe Junoniini (Lepidoptera: Nymphalidae). We show that forewing shape and eyespot size both vary seasonally and that the methods by which phenotype elements change in the dry season forms are different in different clades and may therefore have independent and diverse evolutionary origins.
\end{abstract}

Keywords Polyphenism - Seasonal polyphenism - Shape polyphenism • Color pattern • Pattern evolution • Pattern element $\bullet$ Junoniini $\bullet$ Junonia $\bullet$ Precis

\subsection{Introduction}

Seasonal polyphenism in insects is the phenomenon whereby multiple phenotypes can arise from a single genotype depending on environmental conditions during development. Brakefield and Shapiro more formally define seasonal polyphenism

\footnotetext{
J.W. Clarke (ه)

Department of Biology, Duke University, Durham, NC 27705, USA

e-mail: jameson.clarke@duke.edu 
as the expression of a repeating pattern of changing phenotypes under the control of some environmental factor (Brakefield et al. 1996). Many butterflies have multiple generations per year, and environmentally induced variation in wing color pattern allows them to develop adaptations to the specific season in which the adults live. For example, when predators and prey are both plentiful during the spring or wet season, large striking eyespots may serve to deter or deflect predators (Prudic et al. 2015). In contrast, during the autumn or dry season when prey are scarce, having large striking eyespots might increase the chances of being detected by a predator, and benefit may be obtained by a more cryptic coloration (Brakefield and Larsen 1984).

Because it is potentially adaptive to have specialized forms for predictable environmental heterogeneity, seasonal polyphenism is not uncommon and is most often seen in families Hesperiidae, Lycaenidae, Pieridae, and of course Nymphalidae (Brakefield and Larsen 1984). The elements of butterfly color patterns are developmentally semiautonomous allowing for detailed developmental and evolutionary changes in the overall color pattern (Nijhout 1991). This developmental flexibility of the color pattern can result in extremely diverse seasonal phenotypes within and among species. Seasonal forms of some species, such as Precis octavia, can be so different that they were thought to be a distinct species prior to laboratory experiments that demonstrated that alternative color patterns could be induced by rearing the larvae under varying conditions of temperature and photoperiod (McLeod 1968).

Although the genetic, developmental, and hormonal control of seasonal polyphenism are becoming increasingly understood, there are relatively few studies that examine the evolution of the pattern elements of seasonal forms (Rountree and Nijhout 1995; Monteiro et al. 2015; Oostra et al. 2011). Therefore we asked the following questions: (a) How do wing phenotype elements such as shape and pattern differ between seasonal forms? (b) Can this variation be explained phylogenetically? (c) If so, what are the various pattern strategies used to achieve crypsis in the dry season form? To answer these questions, we analyzed pattern element variation of 34 seasonally polyphenic butterfly species belonging to tribe Junoniini.

The tribe Junoniini (Lepidoptera: Nymphalidae: Nymphalinae) is one of six major tribes in the subfamily Nymphalinae and is comprised of 85 species in 6 genera: Hypolimnas (26 spp.), Precis (17 spp.), Salamis (3 spp.), Yoma (2 spp.), Protogoniomorpha (2 spp.), and Junonia (35 spp.) (Kodandaramaiah 2009). The tribe is estimated to have evolved approximately $30-40$ million years ago originating in Africa and spreading throughout Asia and Oceania primarily (Wahlberg et al. 2005; Kodandaramaiah and Wahlberg 2007). Most of the genera diverged approximately 25 million years ago with the exception of Yoma and Protogoniomorpha which split approximately 5 million years later (2006). Species belonging to this tribe are noted for being swift fliers, having medium to large body sizes, and exhibiting striking polyphenic forms such as the model organism Junonia coenia making the tribe an ideal target taxon for this study (Win et al. 2016).

We show that forewing shape and eyespot size both vary seasonally in the Junoniini and that the methods by which phenotype elements change in the dry 
season forms are different in different clades and may therefore have independent and diverse evolutionary origins.

\subsection{Methods}

Phenotypic variation in the seasonal forms of the Junoniini was measured and assessed using high-resolution digital images and image processing software. Specimens were acquired from the Museum of Natural History in London and the Smithsonian National Museum of Natural History. Images received from the London Museum were on $35 \mathrm{~mm}$ photographic film and were digitized using an EPSON Perfection V600 flatbed digital scanner. Images of the Smithsonian specimens were captured at a fixed distance from the specimen using a Nikon CoolPix P600 16.1 megapixel digital camera. All images were taken with a millimeter ruler for scale.

Specimens were selected for analysis to maximize phylogenetic coverage of tribe Junoniini by including representatives from each of the major genera (Hypolimnas, Junonia, Precis, Protogoniomorpha, Salamis, and Yoma) as well as two out-groups (Anartia and Kallimoides). Selected Junoniini specimens were labeled and organized into the following functional groups corresponding to major clades within the tribe's phylogeny: upper Junonia, Asian Junonia, lower Junonia, Yoma, Precis, and Hypolimnas (Fig. 13.1a). The lower Junonia was treated as a clade for comparative purposes despite its status as an unresolved polytomy. Species were selected that had at least four replicates of comparable quality. To eliminate confounding effects of regional variation and sexual dimorphism, specimens were sexed and selected from the same geographic region. If the species had distinct seasonal phenotypes, these were confirmed by the date of collection and comparison to previous descriptions in the literature. The resulting sample was comprised of 34 species from Africa, Asia, Oceania, and North America.

Size and position of pattern elements of the ventral hind wing were measured by generating landmark points in the image processing software ImageJ v1.51g (Schneider et al. 2012). The ventral surface was chosen because butterflies most often have their wings folded showing the ventral pattern when not in flight. The hind wing was chosen to maximize the amount of visible wing surface since it overlaps in front of the forewing when viewed from the ventral side of the animal. For each image, landmark distances were converted from pixels to millimeters using a $20 \mathrm{~mm}$ scale. To normalize the size and relative distances between pattern elements according to the size of the wing on which they appear, a proxy for wing size was generated using the perimeter of a triangle formed using the distances between the root node of the venation system, the $\mathrm{Cu} 2$ vein terminus, and the $\mathrm{Rs}$ vein terminus, abbreviated RCR triangle (Fig. 13.1b-e).

For the Sc+R1, Rs, M1, M2, M3, and Cu1 cells of the ventral hind wing, the following measurements were taken: 


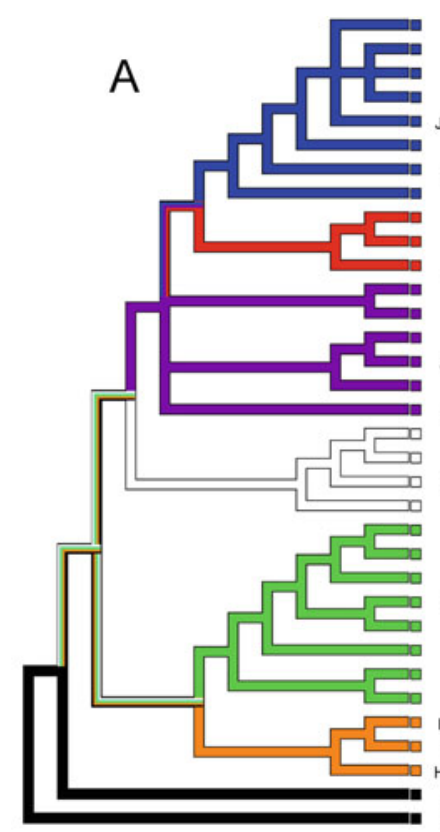

Junonia coenia Junonia orithya Junonia hierta Junonia oenone nonia westermann Junonia villida Junonia lemonias Junonia almana Junonia atlites Junonia iphita Junonia hedonia Junonia natalica Junonia terea Junonia artaxia unonia touhilimasa Junonia cytora Junonia cymodoce Yoma sabina Yoma algina Protog parhassus Salamis cacta Precis cuama Precis tugela Precis sinuata Precis andremiaja Precis antilope Precis ceryne Precis octavia Precis archesia Hypolimnas alimena Hypolimnas bolina Hypolimnas anthedon Anartia amathea Kallimoides rumia

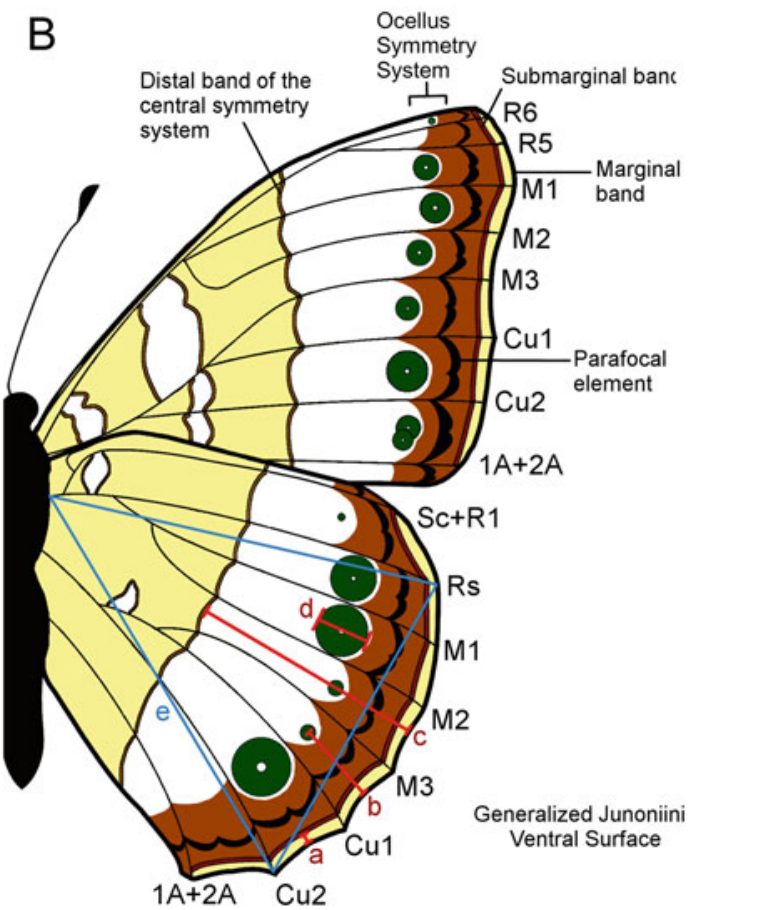

Fig. 13.1 (a) Phylogeny of the Junoniini (Lepidoptera: Nymphalidae: Nymphalinae) species used in this study grouped by reference clade. Tree topology based on Kodandaramaiah and Wahlberg (2007). (b) Diagram of measurements on a generalized Junoniini ventral wing surface including (a) submarginal band proximity [NSP], (b) eyespot proximity [NEP], (c) central symmetry system 


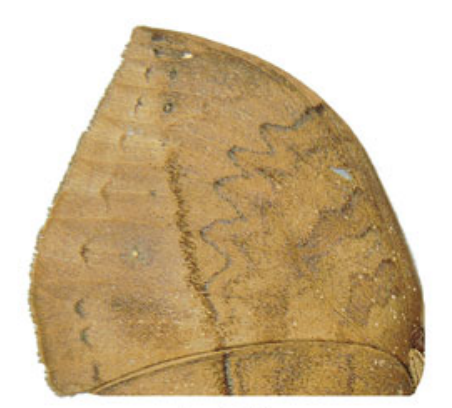

Leaf-like

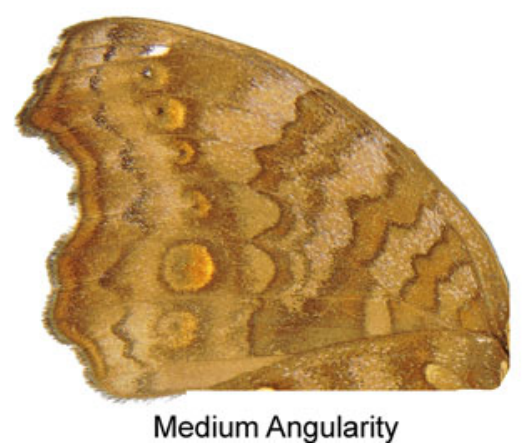

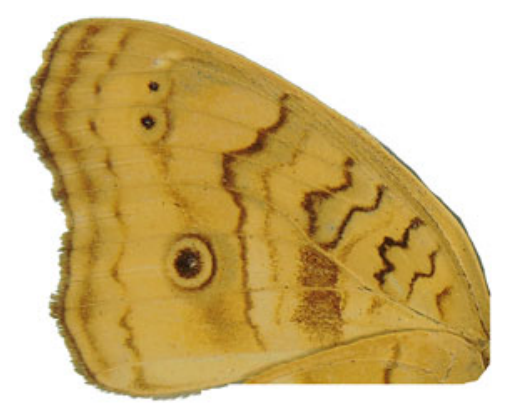

Low Angularity

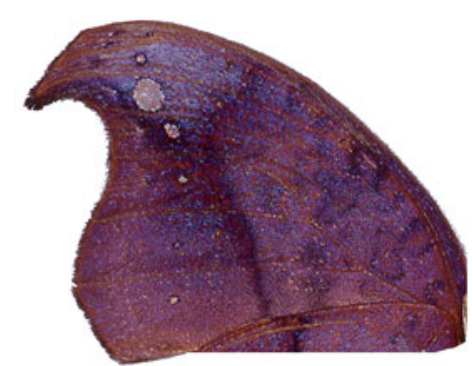

High Angularity

Fig. 13.2 Qualitative categorization of four forewing apex shape classifications observed in the dry season form. Note the increasing acuteness of the angle formed by the margins immediately flanking the M1 terminus as the apex increases in angularity from low to high. Note also that the M1 terminus angle is not present in the leaflike morphotype

(a) [NSP] normalized submarginal band proximity - the distance between the wing margin and the submarginal band divided by the RCR triangle (Fig. 13.1b-a)

(b) [NEP] normalized eyespot proximity - the distance between the wing margin and the focus of the eyespot divided by the RCR triangle (Fig. 13.1b - b)

(c) $[\mathrm{NCP}]$ normalized central symmetry system proximity - the distance between the wing margin and the distal band of the central symmetry system divided by the RCR triangle (Fig. $13.1 \mathrm{~b}-\mathrm{c}$ )

(d) [NED] normalized eyespot diameter - the longest distance that intersects with the focus of an eyespot between the distal and the proximal borders of the eyespot divided by the RCR triangle (Fig. 13.1b - d)

The morphology of the forewing apex was categorized into four classifications: leaflike, low angularity, medium angularity, and high angularity (Fig. 13.2). To

Fig. 13.1 (continued) proximity [NCP], and $(d)$ eyespot diameter [NED]. Measurements were size-normalized using $(e)$ the perimeter of a triangle connecting the $\mathrm{Rs}$ and $\mathrm{Cu} 2$ termini to the root of the venation system [RCR triangle]. (Tree topology from Kodandaramaiah and Wahlberg 2007) 
ensure the consistency of the shape classifications, specimens were independently classified by an outside researcher and by the authors. Each classification converged on the same subdivision of morphotypes.

All measurements, including absolute differences in measurements for each seasonal form, were entered into a character matrix using the software suite Mesquite version 3.04 (Maddison and Maddison 2015). To contrast pattern element data between seasonal forms, parsimony character state reconstructions were mapped to an existing tree topology based on a molecular phylogeny for tribe Junoniini (Kodandaramaiah and Wahlberg 2007) and mirrored to draw comparisons between seasonal forms.

\subsection{Results}

\subsubsection{Variation by Pattern Element}

Variation in pattern elements was dependent on the type of pattern element being measured. The normalized submarginal band proximity (NSP) to the margin of the wing did not vary significantly for any wing cell between seasonal forms or across clades. The normalized central symmetry system proximity (NCP) to the wing margin and the normalized eyespot proximity (NEP) to the wing margin did not vary significantly for any wing cell between seasonal forms, but did show clear differences between clades. Finally, the normalized eyespot diameter (NED) varied significantly between seasonal forms and also showed clear differences between clades. It should also be noted that it is likely that the parafocal element also varies between seasonal forms, but difficulty in consistently defining the boundaries of this pattern element led to its omission from this study.

\subsubsection{Variation by Wing Cell}

Variation in pattern elements was also dependent on the wing cell in which the pattern elements are located. There was little variation in NED, NSP, NCP, or NEP for wing cells Sc+R1, M2, and M3 between seasonal forms for all clades because these eyespots are typically reduced or absent in ventral hind wings in both the wet and dry season forms. In contrast, the Rs, M1, and $\mathrm{Cu} 1$ wing cells showed significant differences in NED and NCP across clades, but only NED varied significantly between seasonal forms. Junonia almana (Fig. 13.3) provides a clear example of the differences in seasonal eyespot size variation across wing cells. In this species, the Sc+R1 eyespot is absent in both seasonal forms. The M2 and M3 eyespots are absent in the wet season form, but are present and highly reduced in the 
Fig. 13.3 Junonia almana seasonal forms. (Left) Wet season form exhibiting regions of high color contrast, large welldefined eyespots, and a low-angularity apex shape. (Right) Dry season form exhibiting low color contrast throughout the entire wing, drastically reduced eyespots, and a high-angularity forewing apex shape

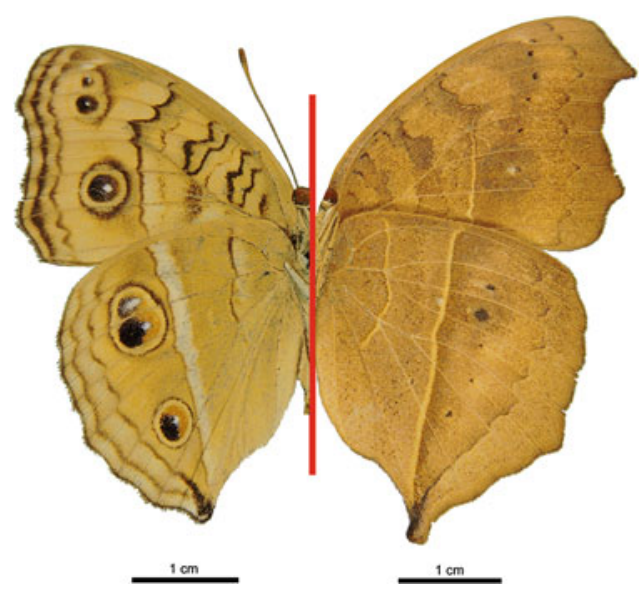

dry season form. Finally, the Rs, M1, and Cu1 eyespots are large in the wet season form and highly reduced in the dry season form (Fig. 13.3).

\subsubsection{Seasonal Eyespot Variation by Clade}

Parsimony analysis of seasonal eyespot variation resulted in three similar parsimony character state reconstructions for the Rs, M1, and $\mathrm{Cu} 1$ eyespots when mapped to the molecular phylogeny. (Figs. 13.4, 13.5, and 13.6). However, the seasonal eyespot variation for each wing cell was not consistent across clades.

All species in the upper Junonia clade exhibited high seasonal eyespot size variation for the Rs, M1, and $\mathrm{Cu} 1$ eyespots with two exceptions: J. westermanni has no variation in the M1 eyespot (Fig. 13.5) and J. hierta in the Cu1 eyespot (Fig. 13.6).

The lower Junonia clade varied greatly both across species and by wing cell for seasonal eyespot size. For the Rs eyespot, most species showed no seasonal size variation except slightly in J. cytora and J. touhilimasa (Fig. 13.4). However, for the M1 and Cu1 eyespots, all of the species showed some seasonal size variation, though to different degrees, with the single exception of J. cytora in the Cu1 cell (Figs. 13.5 and 13.6).

The Yoma clade also exhibited a wide range of seasonal eyespot size variation. Yoma algina showed large seasonal eyespot size variation for the Rs, M1, and Cu1 eyespots, while its sister taxon $Y$. sabina showed only minimal variation in the Rs and M1 eyespots and no variation in the $\mathrm{Cu} 1$ eyespot. Protogoniomorpha parhassus exhibited seasonal eyespot size variation in the M1 and $\mathrm{Cu} 1$ eyespots but not the Rs eyespot. Finally, Salamis cacta showed no seasonal eyespot size variation whatsoever (Figs. 13.4, 13.5, and 13.8). 

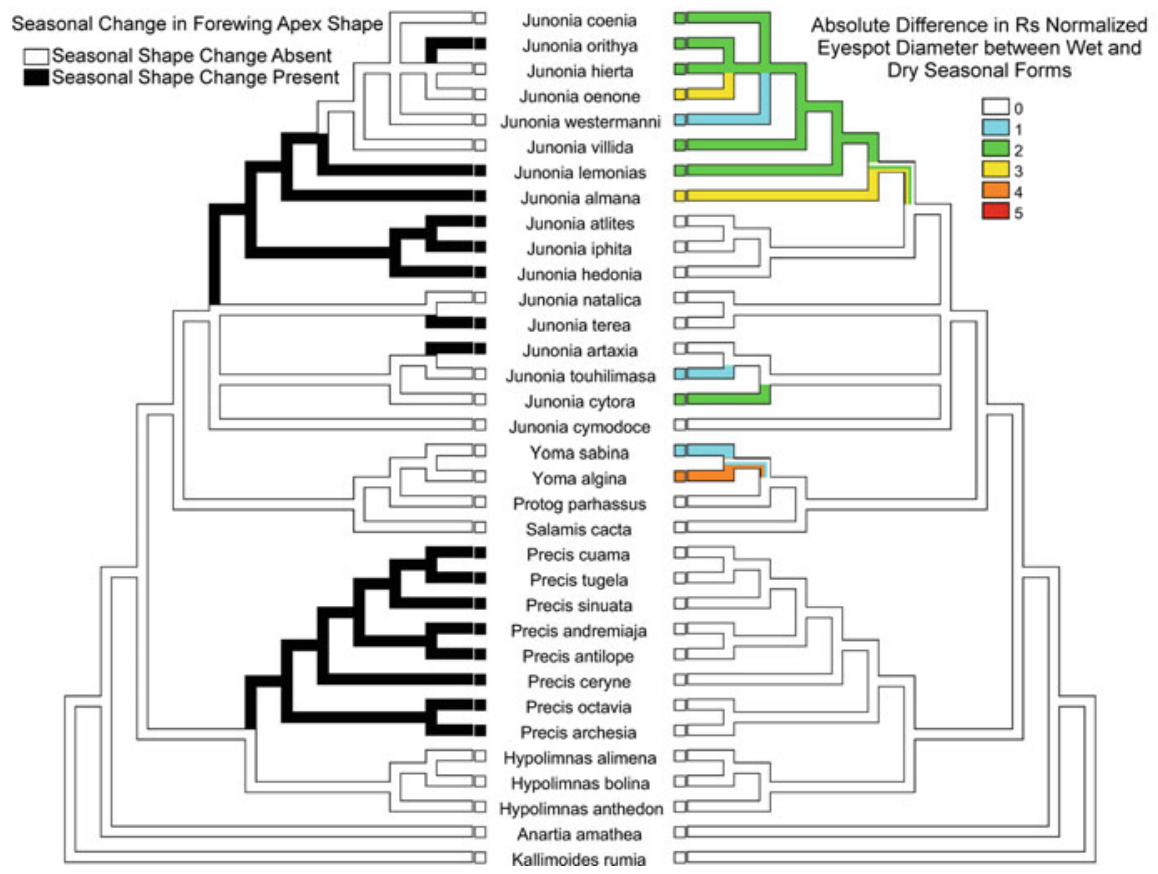

Fig. 13.4 Mirrored parsimony character state reconstructions for seasonal change in forewing apex shape class and the absolute difference in Rs normalized eyespot diameter between wet and dry season forms. Warmer colors represent a larger disparity between eyespot diameters of wet and dry forms (Tree topology from Kodandaramaiah and Wahlberg 2007)

Finally, the Precis clade, the Hypolimnas clade, and the Asian Junonia showed almost no seasonal eyespot size variation for any eyespot with one exception in $H$. anthedon which had the unusual characteristic of having no eyespots in (Figs. 13.4, 13.5, and 13.6).

\subsubsection{Seasonal Forewing Apex Shape Change by Clade}

The shape of the forewing apex varied both seasonally and by clade. The upper Junonia clade exhibited a mix of seasonal variation with shape change present only in J. orithya, J. lemonias, and J. almana. Similarly, in the lower Junonia clade, only two species, J. terea and J. artaxia, showed seasonal shape change of the forewing. The remaining clades, however, do not have this mix of shape change between seasonal forms. Neither the Yoma nor the Hypolimnas clades showed seasonal shape change. In contrast, both the Asian Junonia and Precis clades showed seasonal shape change in every species sampled (Fig. 13.7). 

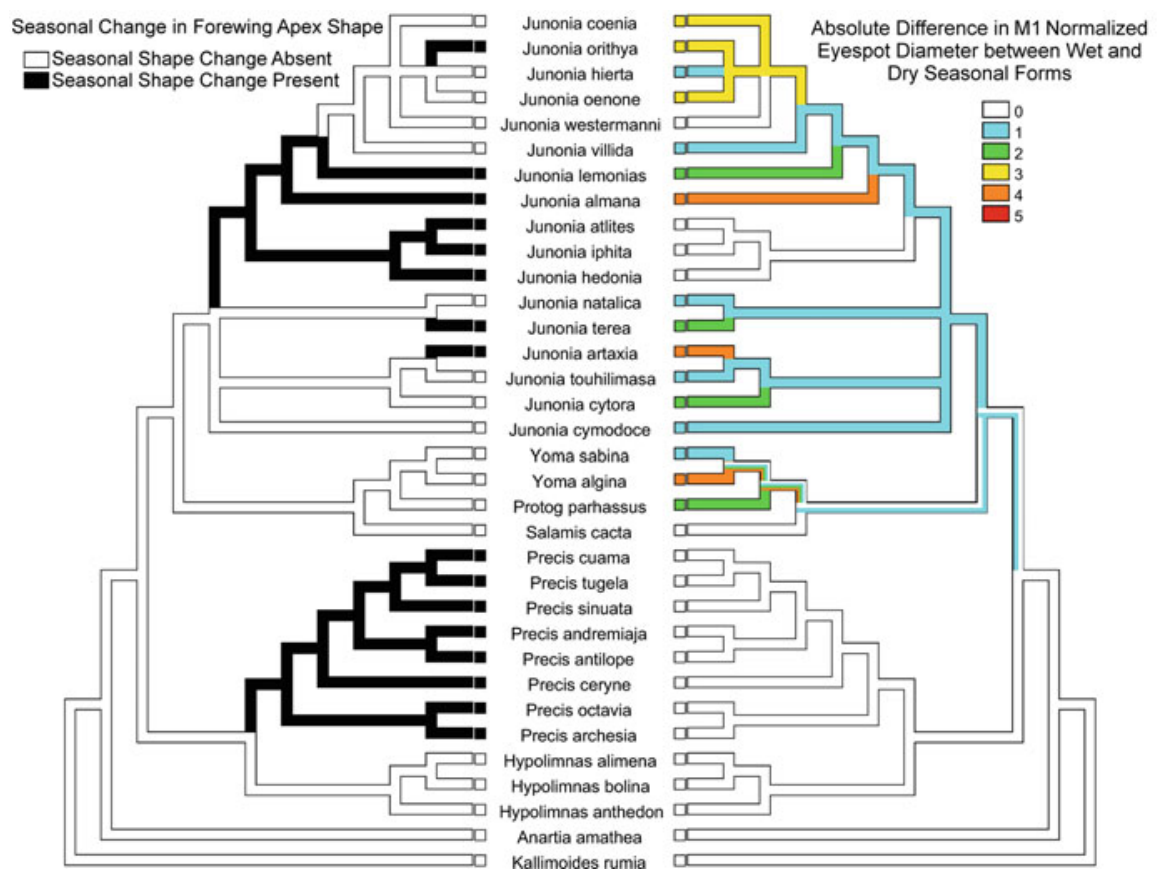

Fig. 13.5 Mirrored parsimony character state reconstructions for seasonal change in forewing apex shape class (left) and the absolute difference in M1 normalized eyespot diameter between wet and dry season forms (right). Warmer colors represent a larger disparity between eyespot diameters of wet and dry forms (Tree topology from Kodandaramaiah and Wahlberg 2007)

\subsubsection{Shape Type and Shape Change}

There exists an association between the shape of the forewing apex of the dry season form of a species and whether or not there was seasonal shape variation for that species. Species whose dry season form had a low angularity or leaflike forewing apex invariably did not exhibit seasonal forewing shape change (Fig. 13.7). Furthermore, species whose forewing apex varied seasonally did so according to a pattern of increasing angularity in the dry season form compared to the wet season form (Figs. 13.7 and 13.8 - bottom). Species with seasonal shape change whose wet season form had a low-angularity forewing apex had dry season forms with medium- or high-angularity forewing apex shapes. Similarly, species with seasonal shape change whose wet season form had a medium-angularity forewing apex had dry season forms with high-angularity forewing apex shapes. The result of this association is the general trend that species that exhibit seasonal change in forewing shape tend to have more high-angularity forewing morphologies, while species that do not exhibit seasonal change tend to have low-angularity forewing morphologies. 

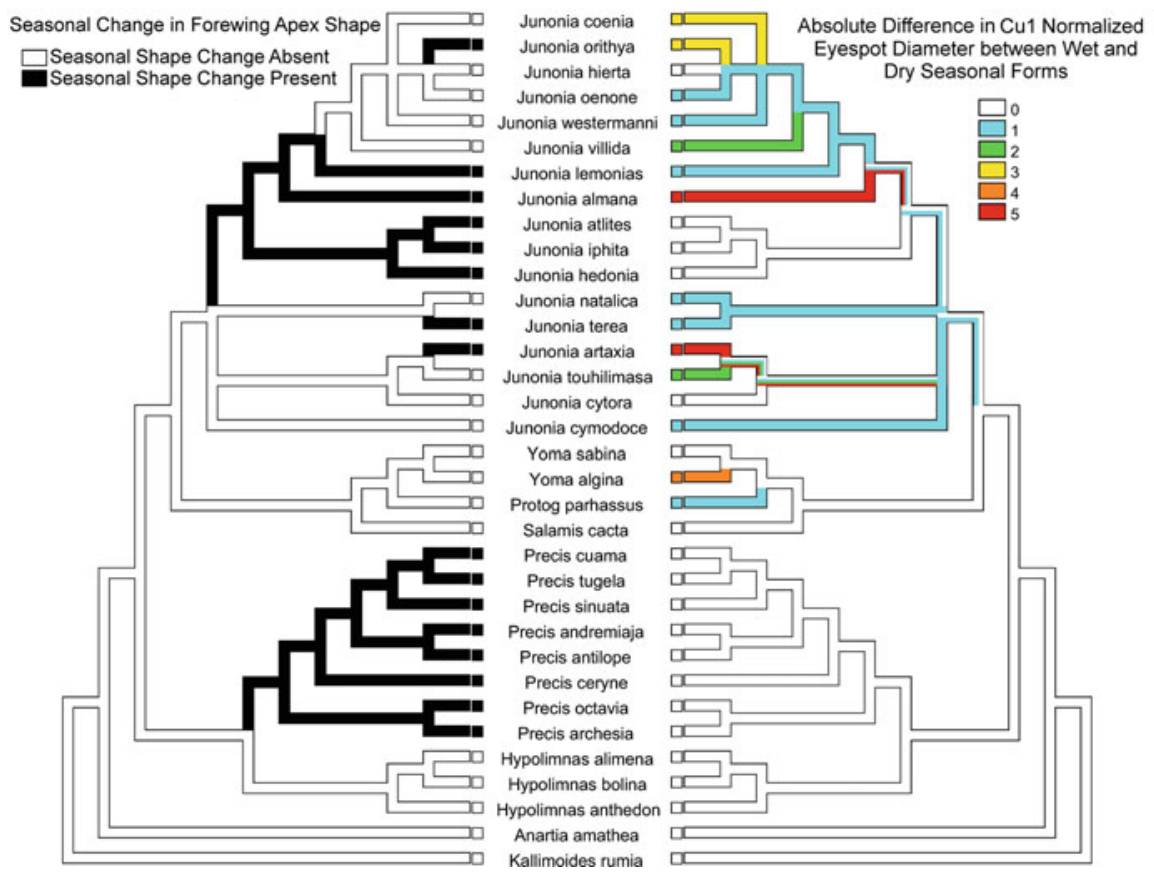

Fig. 13.6 Mirrored parsimony character state reconstructions for seasonal change in forewing apex shape class (left) and the absolute difference in Cu1 normalized eyespot diameter between wet and dry season forms (right). Warmer colors represent a larger disparity between eyespot diameters of wet and dry forms (Tree topology from Kodandaramaiah and Wahlberg 2007)

\subsubsection{Discussion}

The results of this investigation reveal a dynamic relationship between the components of a seasonally polyphenic phenotype and the pattern by which they evolve. They show that the phenotype elements of a seasonal form can be evolutionarily decoupled, that these plastic elements have responded to selective pressures in diverse ways, and that the diversity of these responses is reflected in the tribe's phylogenetic history. The different pattern elements of seasonally polyphenic forms do not evolve as a single cohesive unit.

The methods by which the seasonal forms have evolved in these butterflies also offer insight about how pattern elements are involved in responding to selection for crypsis in the dry season. The findings of this study suggest that the location of pattern elements on the wing does not change in the formation of alternative seasonal phenotypes. This suggests that either selection is not acting strongly on position or that element position is under some developmental constraint and therefore unable to respond to selective pressures. When considering the variation in the position of eyespots specifically, it seems more likely to be a lack of selection on position given that eyespot position is characteristically different among clades, 

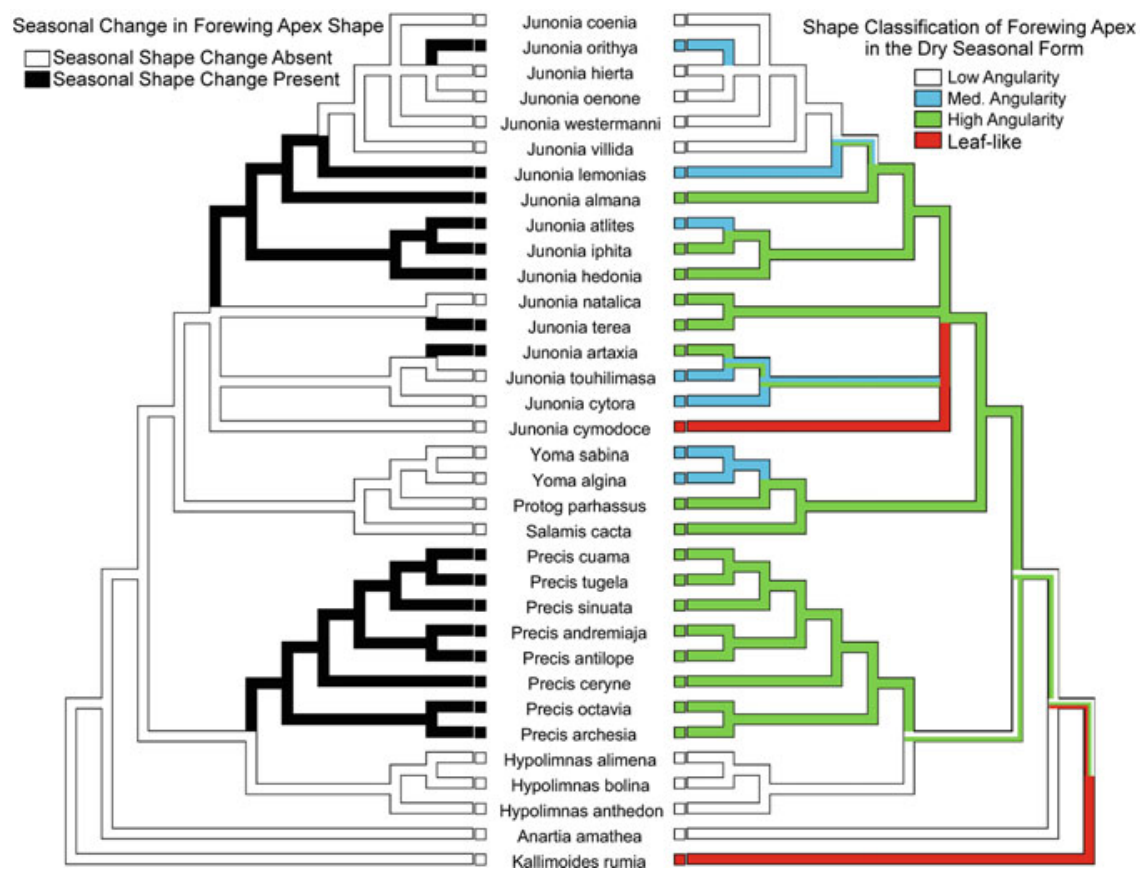

Fig. 13.7 Mirrored parsimony character state reconstructions for seasonal change in forewing apex shape class and the shape classification of the forewing apex in the dry season form. Note the association between exhibiting seasonal shape change and having higher forewing apex angularity in the dry season form (Tree topology from Kodandaramaiah and Wahlberg 2007)

implying at least some freedom from constraint during the early evolution of the major Junoniini clades.

However, the variation in eyespot size tells us a different story. In species where eyespot size is variable, the dry season form always has reduced eyespot size compared to the wet season form, suggesting a strong response to selection. At the same time, there is little variation in the wing cells in which large eyespots develop. For instance, the upper and lower Junonia clades all have stable large eyespots in the Rs, M1, and Cu1 wing cells and stable small eyespots in the M2 and M3 wing cells in the wet season form, whereas species in the Precis clade have stable small eyespots in all of the wing cells. There is no case where stable large eyespots have evolved in the M2 and M3 wing cells. Thus there appear to be constraints both on the capacity to have size-variable eyespots and on the wing cell in which the eyespot is found (Figs. 13.4 and 13.8 - bottom).

Similarly, in clades that have seasonal variation in wing shape, the dry season form always has a more angular or falcate forewing shape, again suggesting a strong response to selection (Figs. 13.7 and 13.8 - bottom). Other clades, by contrast, have evolved phenotypically stable low-angularity rounded (Hypolimnas clade) or high-angularity falcate (Yoma and Asian Junonia clades) wing shapes. 

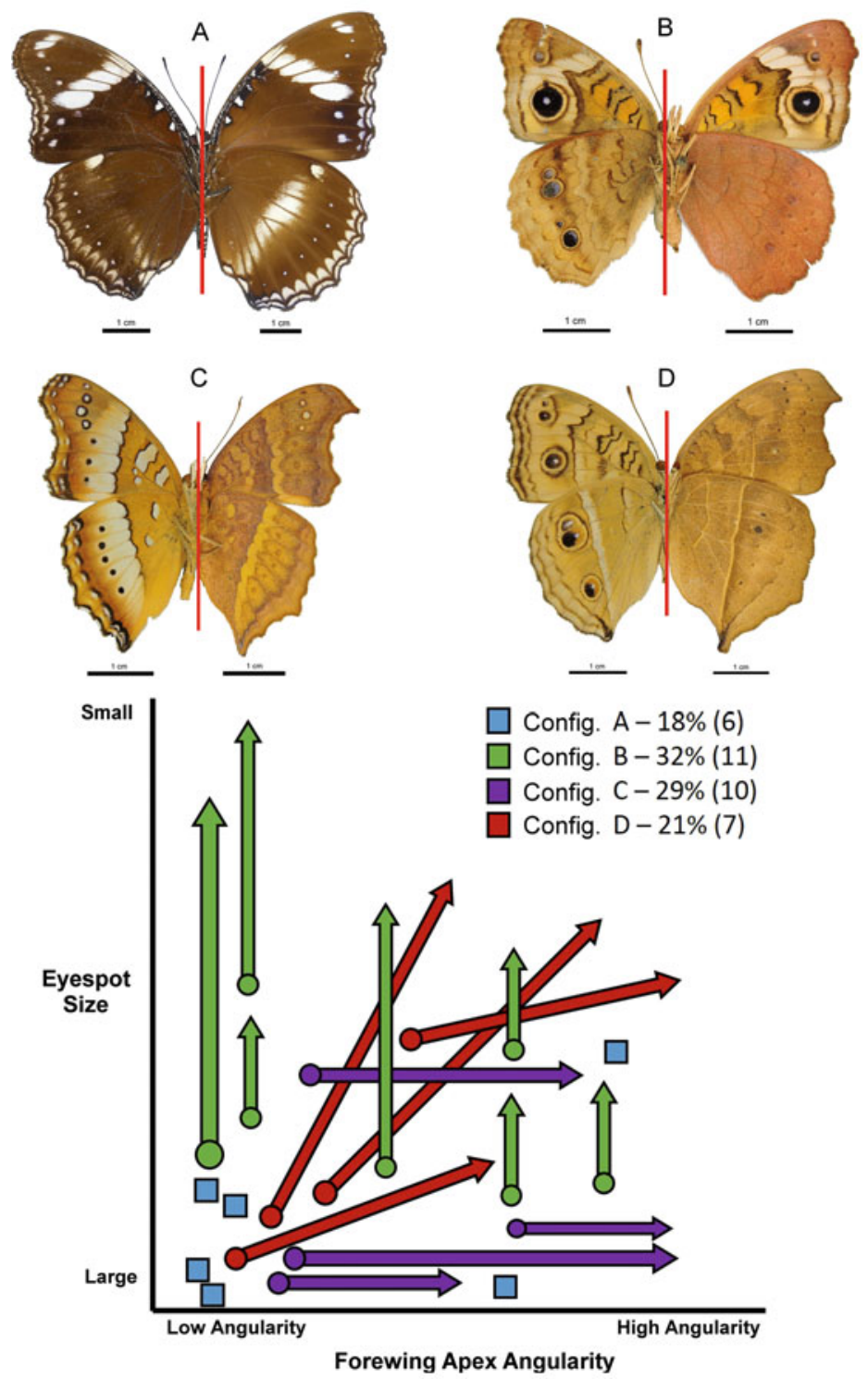

Fig. 13.8 Top: Plates representing four configurations found among Junoniini butterflies - (a) Neither forewing shape nor eyespot size vary seasonally; (b) Only eyespot size varies seasonally; (c) Only forewing shape varies seasonally; (d) Both forewing shape and eyespot size vary seasonally. Each plate is a composite of the wet season form (left) and the dry season form (right). Bottom: Schematic diagram of the relationship between seasonal changes in forewing shape and eyespot size. Wet season forms are shown as circles, dry season forms as arrows, and when the two forms overlap they are shown as squares. Note that the change for forewing shape is always from lower to higher angularity, and the change in eyespot size is always a reduction 
Thus the Junoniini show both plastic alternative wing shape phenotypes and evolutionary fixation of the alternative shapes, and the fixation of either the lowor high-angularity wing shapes corresponds to major clade divergences in the phylogeny. This suggests that while the capacity to develop alternative wing shapes evolved early for the entire tribe, the fixation of alternative wing shapes occurred later in the establishment of the genera.

The decoupling of independent phenotype elements has allowed for the emergence of what we loosely refer to here as "phenotypic configurations" which can be thought of as arrangements of phenotype element variation. To illustrate this idea, we used only the combination of eyespot size variation and forewing apex shape variation which can be roughly categorized into the four configurations that represent butterflies whose seasonal forms exhibit no variation, size variation only, shape variation only, or both size and shape variation: Configurations $\mathrm{A}, \mathrm{B}, \mathrm{C}$, and D, respectively (Fig. 13.8 - top).

The distribution of these configurations in the phylogeny shows some interesting patterns. First, each of the configurations corresponds to the clades described in Fig. 13.1 - top: the Hypolimnas clade shows almost no seasonal change in wing shape or eyespot size (Figs. 13.4 and 13.8 - top: Configuration A); the upper Junonia exhibit minimal seasonal change in wing shape, but exhibit great seasonal change in eyespot size (Figs. 13.4 and Fig. 13.8 - top: Configuration B); the inverse is true for the Precis and Asian Junonia clades whose members show almost no seasonal change in eyespot size, while they all exhibit seasonal change in forewing apex shape (Figs. 13.4 and Fig. 13.8 - top: Configuration C). Second, the relative frequency of these configurations and their position in the phylogeny show that species whose wing shape varies seasonally tend not to be the same species whose eyespot size varies seasonally, with a few exceptions. A highly angular wing shape in the dry season form seems to have evolved very early in the tribe but was lost independently in both Hypolimnas and upper Junonia (Fig. 13.4 right). Interestingly, the capacity to develop plastic seasonally distinct wing shapes may have evolved around the same time as the falcate wing shape but saw successive loss in Hypolimnas, Yoma, and some species in lower and upper Junonia (Fig. 13.4 left). An alternative interpretation might be that the capacity for wing shape change evolved multiple times - once in the Precis clade and once in genus Junonia with only the latter having some subsequent loss of the trait (Fig. 13.4 left).

Finally, the leaflike wing shape seems to have evolved independently with respect to the well-known leaf mimics of the genus Kallimoides. This raises some interesting questions regarding the importance of the phenotypic elements for crypsis. If a species can achieve crypsis by either reducing its eyespots or changing its wing shape from season to season, why do one or the other? Is the plasticity of one phenotype element enough to render the plasticity of another unnecessary? What, then, of species who have variation in both or neither of these elements?

Another interesting question regarding the interplay between phenotypic elements and their evolutionary trajectory is the role of color and contrast of the wing pattern. In the same way, the conspicuousness of an eyespot can be diminished by reducing its size; it can also be recolored or recontrasted to match the surrounding 
region of the wing, which effectively achieves the same result. This is the case in Precis atlites, whose eyespots remain the same in size but become less bold and more similar in color to the background of the wing rendering them more difficult to detect. Although seasonal changes in color and contrast are widespread in the tribe, that is to say all of the dry season forms become duskier in color and less striking in the boldness of their patterns, it is unclear as to what extent changing color and contrast compensate for the inability to modify either wing shape or eyespot size. A detailed analysis of these elements will be presented in a separate paper.

The seasonally polyphenic forms of butterflies are often thought of as a single trait. In reality, because butterfly wing patterns are comprised of serially homologous phenotypic elements that are developmentally semiautonomous, they can be uncoupled, modified, and reconfigured to respond to selection and produce constraints in diverse ways. The seasonal forms of Junoniini butterflies have changed over time by invoking at least three distinct developmental mechanisms, including wing shape morphogenesis, pigment synthesis pathways, and pattern element positioning mechanisms. Rather than inheriting a seasonal form, these butterflies inherit the tools to create a seasonal form, and the methods by which they have convergently evolved to become cryptic are written in their evolutionary history.

Acknowledgments I am grateful to the Smithsonian Institution National Museum of Natural History and the National History Museum in London for access to their butterfly collections, Professor H.F. Nijhout for his direction and guidance, Richard Gawne and Kenneth McKenna for their helpful discussion and criticism, Leo Kerner for assistance in image databasing, the anonymous reviewers for their criticism and feedback, and the support of the following National Science Foundation grants awarded to H.F. Nijhout: IOS-0641144, IOS-1557341, and IOS-1121065.

\section{References}

Brakefield PM, Larsen TB (1984) The evolutionary significance of dry and wet season forms in some tropical butterflies. Biol J Linnean Soc 22(1):1-12

Brakefield PM, Gates J, Keys D, Kesbeke F, Wijngaarden PJ, Monteiro A, French V, Carroll SB (1996) Development, plasticity and evolution of butterfly eyespot patterns. Nature 384 (6606):236-242

Kodandaramaiah U (2009) Eyespot evolution: phylogenetic insights from Junonia and related butterfly genera (Nymphalidae: Junoniini). Evol Dev 11(5):489-497

Kodandaramaiah U, Wahlberg N (2007) Out-of-Africa origin and dispersal-mediated diversification of the butterfly genus Junonia (Nymphalidae: Nymphalinae). J Evol Biol 20(6):2181-2191

Maddison WP, Maddison DR (2015) Mesquite: a modular system for evolutionary analysis. Version 3.04. http://mesquiteproject.org

McLeod L (1968) Controlled environment experiments with Precis octavia Cram (Nymphalidae). J Res Lepidoptera 8(2):53-54

Monteiro A, Tong X, Bear A, Liew SF, Bhardwaj S, Wasik BR, Dinwiddie A, Bastianelli C, Cheong WF, Wenk MR, Cao H (2015) Differential expression of ecdysone receptor leads to variation in phenotypic plasticity across serial homologs. PLoS Genet 11(9):e1005529

Nijhout HF (1991) The development and evolution of butterfly wing patterns, Smithsonian series in comparative evolutionary biology. Smithsonian Institution Press, Washington 
Oostra V, de Jong MA, Invergo BM, Kesbeke F, Wende F, Brakefield PM, Zwaan BJ (2011) Translating environmental gradients into discontinuous reaction norms via hormone signalling in a polyphenic butterfly. Proc R Soc Lond B Biol Sci 278(1706):789-797

Prudic KL, Stoehr AM, Wasik BR, Monteiro A (2015) Eyespots deflect predator attack increasing fitness and promoting the evolution of phenotypic plasticity. Proc R Soc B 282 (1798):20141531. The Royal Society

Rountree DB, Nijhout HF (1995) Genetic control of a seasonal morph in Precis coenia (Lepidoptera: Nymphalidae). J Insect Physiol 41(12):1141-1145

Schneider CA, Rasband WS, Eliceiri KW (2012) NIH Image to ImageJ: 25 years of image analysis. Nat Method 9(7):671-675

Wahlberg N (2006) That awkward age for butterflies: insights from the age of the butterfly subfamily Nymphalinae (Lepidoptera: Nymphalidae). Syst Biol 55(5):703-714

Wahlberg N, Brower AV, Nylin S (2005) Phylogenetic relationships and historical biogeography of tribes and genera in the subfamily Nymphalinae (Lepidoptera: Nymphalidae). Biol J Linnean Soc 86(2):227-251

Win NZ, Choi EY, Park J, Park JK (2016) Taxonomic review of the tribe Junoniini (Lepidoptera: Nymphalidae: Nymphalinae) from Myanmar. J Asia-Pacific Biodiv 9(3):383-388

Open Access This chapter is licensed under the terms of the Creative Commons Attribution 4.0 International License (http://creativecommons.org/licenses/by/4.0/), which permits use, sharing, adaptation, distribution and reproduction in any medium or format, as long as you give appropriate credit to the original author(s) and the source, provide a link to the Creative Commons license and indicate if changes were made.

The images or other third party material in this chapter are included in the chapter's Creative Commons license, unless indicated otherwise in a credit line to the material. If material is not included in the chapter's Creative Commons license and your intended use is not permitted by statutory regulation or exceeds the permitted use, you will need to obtain permission directly from the copyright holder.

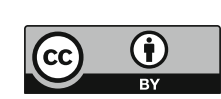

